\title{
STOCHASTIC MODAL MODELS OF SLENDER UNCERTAIN CURVED BEAMS PRELOADED THROUGH CLAMPING
}

\author{
Javier Avalos ${ }^{1}$, Lanae A. Richter ${ }^{2}$, X.Q. Wang ${ }^{2}$, Raghavendra Murthy ${ }^{2}$, \\ and Marc P. Mignolet ${ }^{2 *}$ \\ ${ }^{1}$ Departamento de Procesos Tecnológicos e Industriales \\ Instituto Technológico y de Estudios Superiores de Occidente (ITESO) \\ Tlaquepaque, Jalisco, Mexico 45604 \\ ${ }^{2}$ SEMTE, Faculties of Mechanical and Aerospace Engineering \\ Arizona State University \\ Tempe, Arizona 85287-6106, USA
}

\section{ABSTRACT}

This paper addresses the stochastic modeling of the stiffness matrix of slender uncertain curved beams that are forced fit into a clamped-clamped fixture designed for straight beams. Because of the misfit with the clamps, the final shape of the clamped-clamped beams is not straight and they are subjected to an axial preload. Both of these features are uncertain given the uncertainty on the initial, undeformed shape of the beams and affect significantly the stiffness matrix associated with small motions around the clamped-clamped configuration. A modal model using linear modes of the straight clamped-clamped beam with a randomized stiffness matrix is employed to characterize the linear dynamic behavior of the uncertain beams. This stiffness matrix is modeled using a mixed nonparametric-parametric stochastic model in which the nonparametric (maximum entropy) component is used to model the uncertainty in final shape while the preload is explicitly, parametrically included in the stiffness matrix representation. Finally, a maximum likelihood framework is proposed for the identification of the parameters associated with the uncertainty level and the mean model, or part thereof, using either natural frequencies only or natural 
frequencies and mode shape information of the beams around their final clamped-clamped state.

To validate these concepts, three simulated, computational experiments were conducted within Nastran to produce populations of natural frequencies and mode shapes of uncertain slender curved beams after clamping. The three experiments differed from each other by the nature of the clamping condition in the in-plane direction. One experiment assumed a no-slip condition (zero in-plane displacement), another a perfect slip (no in-plane force), while the third one invoked friction. The first two experiments gave distributions of frequencies with similar features while the latter one yielded a strong deterministic dependence of the frequencies on each other, a situation observed and explained recently and thus not considered further here. Then, the application of the stochastic modeling concepts to the no-slip simulated data was carried out and led to a good matching of the probability density functions of the natural frequencies and the modal components, even though this information was not used in the identification process. These results strongly suggest the applicability of the proposed stochastic model.

KEYWORDS: uncertain geometry, uncertain preload, uncertain stiffness, maximum entropy, parametric stochastic model

\section{INTRODUCTION}

The assembly of substructures is well known to induce uncertainty on the dynamic behavior of the overall structure they form, e.g. see [1] for a comprehensive review and [2,3] for recent studies. The flexibility of the attachment has in particular been recognized as a notable source of this uncertainty and both parametric, e.g. see [4,5], and maximum entropy based nonparametric [6,7] stochastic models of the corresponding stiffness properties have been proposed. The latter 
method, see [8] for a recent state-of-the-art is particularly convenient as it can also incorporate, as demonstrated in $[9,10]$, many other model and data uncertainties that exist in attachment modeling, e.g. contact and friction/microslip nonlinearities, variability in friction properties and loading induced by fasteners.

An uncertainty which appears to have received little attention so far is the one induced by the random state of stress, referred to as preload in the sequel, that tends to arise during the assembly due to a slight mismatch in shape or size of a substructure as compared to the ones it should have to fit perfectly in the assembly. When the substructure considered is very slender/thin, an axial state of stress induces a large change in natural frequencies, especially for the lowest ones while the change in mode shape may be significantly less. Clearly then, uncertainty in the preload magnitude and distribution, rooted for example in shape/size mismatch, must be included in the stochastic modal model.

A preliminary effort in this direction was accomplished in [11] as part of a broader investigation focused on the experimental validation of stochastic reduced order models for the geometrically nonlinear response of beams. In this study, 11 nominally straight but effectively curved beams were set in a fixture providing a near clamping on both ends (see section 2 for experimental detail) and their first three natural frequencies were measured experimentally. It was observed that these triplets of frequencies deviated significantly from those of the flat beam as well as from one another, i.e. with standard deviations equal to $25 \%, 12 \%$, and $7 \%$ of the flat beam values for the first, second, and third natural frequencies, respectively. Further, a very strong correlation between the three frequency variations were observed. 
Relying on a parallel between the clamping induced preload and a change in the beam temperature, it was proposed in [11] that the modal stiffness matrix $\mathbf{K}$ of the clamped-clamped beams be expressed as the sum of two terms, i.e. as

$$
\mathbf{K}=\mathbf{K}_{0}+P \mathbf{K}_{1}
$$

where $\mathbf{K}_{0}$ and $\mathbf{K}_{1}$ are the preload independent and preload induced modal stiffnesses with $P$ denoting the preload. A justification of Eq. (1) can be drawn from the analysis of shallow slender beams under preload. Specifically, considering the beam to be straight under the action of a constant preload $P$ and adopting an Euler-Bernoulli model leads to the partial differential equation for the transverse displacements $y(x, t)$

$$
\rho A \frac{\partial^{2} y}{\partial t^{2}}+E I \frac{\partial^{4} y}{\partial x^{4}}-P \frac{\partial^{2} y}{\partial x^{2}}=0
$$

where, following standard notations, $E, \rho, I$, and $A$ are the beam's Young modulus, density, and its cross-section moment of inertia and area. Note in Eq. (2) that the stiffness operator on the lefthand-side is the sum of two operators, the first one, $E I \frac{\partial^{4} y}{\partial x^{4}}$ is independent of the preload $P$ while the second one, $P \frac{\partial^{2} y}{\partial x^{2}}$ is linear in this variable. Thus, a finite element implementation of Eq. (2) leads to a stiffness matrix $\mathbf{K}$ in the form of Eq. (1).

Note as well from Eq. (2) that the geometry of the beam is present in the two operators composing the stiffness. Then, it is tentatively suggested that the two matrices $\mathbf{K}_{0}$ and $\mathbf{K}_{1}$ be considered random, described more specifically by the nonparametric approach, while the preload $P$ would be represented as a random variable independent of $\mathbf{K}_{0}$ and $\mathbf{K}_{1}$. In fact, a Gaussian assumption on $P$ was proposed [11] consistently with the maximum entropy principle. 
However, a validation of this full stochastic model was not performed in [11] because the very strong correlation between the measured natural frequencies suggested that the dominant uncertainty was on the preload, not the matrices $\mathbf{K}_{0}$ and $\mathbf{K}_{1}$, an assumption that was supported by a good fit of the frequency data obtained by selecting those matrices as those associated with the flat beam and an appropriate (least square) selection of the preload values. In fact, a revisit of this issue [12] obtained an even closer fit of the first and third natural frequencies (those best captured in the test) with an appropriate updating of the matrix $\mathbf{K}_{1}$.

In light of these observations, the focus of the present investigation is first on suggesting an explanation for the behavior seen in [11,12] and second on considering in details a related case in which at least one of the two matrices $\mathbf{K}_{0}$ and $\mathbf{K}_{1}$ ought to be randomized. Key in these efforts is the availability of data. To this end, a "computational experiment" is first carried out which models the clamping process of the beams in the original study of [11] but can be performed over a large sample of beams and yields a complete description of displacements, forces, natural frequencies, and mode shapes which would represent a daunting task to capture experimentally. This computational experiment is described in the next section.

\section{THE COMPUTATIONAL EXPERIMENT}

The physical experiment reported in [11] involved 11 precision-machined feeler gages of nominal thickness, width, and length equal to 0.031 in $(0.79 \mathrm{~mm})$, $0.5 \mathrm{in}(1.27 \mathrm{~cm})$, and $12 \mathrm{in}$ $(30.48 \mathrm{~cm})$. These beams were clamped in the fixture shown in Fig. 1(a) through two clamping blocks, one at each end, of length $1.5 \mathrm{in}(3.81 \mathrm{~cm})$ which were screwed in the base of the fixture. Thus, the length of the beam, from clamp to clamp was 9in $(22.86 \mathrm{~cm})$. Further, the material 
properties of the beams were identified in an earlier investigation [13] as: Young's modulus of 204.7 GPa, Poisson’s ratio of 0.28 , and mass density of $7.86 \bullet 10^{3} \mathrm{~kg} / \mathrm{m}^{3}$.

This physical experiment was simulated computationally as described in Fig. 1(b). Initially curved beams of length $L$ equal to 12 in $(30.48 \mathrm{~cm})$ were discretized by finite elements within Nastran using beam elements (referred to as "CBEAM") and 51 nodes spanning the entire beam length. Further, nodes 1-6 and 46-51 were positioned directly under the clamping blocks. Clamping was then simulated by imposing that the vertical (i.e., along y) positions and out-ofplane (i.e., along z) rotations of the nodes 1-6 and 46-51 become zero. Out of plane displacements (i.e., along $z$ ) and rotations (i.e., along $y$ and $x$ ) of these nodes were also assumed to stay zero. A more subtle question regards the in-plane (i.e., along $x$ ) displacements of these nodes. Three particular scenarios were considered here:

(A) a no-slip condition in which all in-plane displacements were imposed to zero,

(B) a perfect slip condition in which all in-plane displacements were free to move, i.e. with zero in-plane force, on one side with the other under a no-slip condition to prevent rigid body motions,

(C) a slip condition with friction in which the in-plane load was limited to $\mu N$ where $N$ is the smallest of the vertical forces at the two clamps.

The numerical clamping, either A, B, or C, of each beam considered was achieved as a nonlinear static problem (the Nastran solution 106 or "SOL 106") which was followed by a linear modal analysis that provided the natural frequencies $\omega_{j}^{(i)}$, mode shapes $\underline{\phi}_{j}^{(i)}$, and linear stiffness matrix $\mathbf{K}_{G}^{(i)}$ of each preloaded beams (superscript (i)) for small motions around their deformed positions. Note that option C was implemented as a two step process. First, a no-slip condition 
was imposed on both clamps and the corresponding in-plane and vertical forces were determined, the smallest of the two, $N$, in particular. If the in-plane load was found to exceed $\mu N$ ( $\mu$ was selected here as 0.3), then the boundary condition on the side exhibiting the smallest vertical force (i.e., $N$ ) was replaced by a perfect slip condition to which was added the force $\mu N$ in the direction of the original in-plane force. The nonlinear static deformed configuration resulting from this second step was the one used for the determination of the natural frequencies and modes.

In keeping with the smooth shape of the experimental beams, the random undeformed profiles of the beams were selected to be of the form

$$
y(x)=\beta_{0} \sin \pi x / L+\beta_{1} \sin 2 \pi x / L+\beta_{2} \sin 3 \pi x / L, \quad x \in[0, L]
$$

where $\beta_{0}, \beta_{1}$, and $\beta_{2}$ were chosen as independent zero mean Gaussian random variables. An inspection of the experimental beams suggested that the peak of the profile be typically less than one thickness ( $h=0.79 \mathrm{~mm})$ and that the profiles be dominated by their half-wave components (i.e. $\beta_{0}$ ). These observations led to the selection of the standard deviations $\sigma_{0}, \sigma_{1}$, and $\sigma_{2}$ of $\beta_{0}$, $\beta_{1}$, and $\beta_{2}$ as $\eta h / 2, \eta h / 4$ and $\eta h / 6$, respectively, with $\eta=1$ for cases $A$ and $B$ and 0.5 for case C.

A population of $M=1000$ such curved beams were simulated and subjected to each of the three computational clamping options described above. Scatter plots of the beam frequencies $f_{1}$, $f_{2}, f_{3}$ are presented in Figs 2 and 3. First and foremost, note that a large fraction of the frequencies obtained in case C, Fig. 2(c), and 3(c) are strongly concentrated around a curve in the $\left(f_{1}, f_{2}, f_{3}\right)$ space indicating that these frequencies depend only on one random parameter, i.e., the preload $\mu N$. This situation is in contrast with cases A and B but in full agreement with the experimental observations of [11], see [12]. These results suggest that a friction-controlled slip is the 
mechanism that induced the preload dominated variations in natural frequencies seen and modeled in these investigations. Since that case has been clarified in [12], it will not be covered in the present investigation. Rather, the focus will be on either of the two other cases for which a wide spread of frequencies exist and for which the stochastic modeling of the preload $P$ will clearly not be sufficient. More specifically, the no-slip case (option A) was considered.

In keeping with the focus of the original study [11] on the dynamic response of the beams in the low frequency range, modal models of the computationally clamped beams were established using the first 10 transverse and first 10 in-plane mass normalized modes of the straight clampedclamped beam. These 20 modes, $\underline{\psi}_{j}$ for $j=1, \ldots, 20$, were stacked as columns in the matrix $\Psi$ and the linear modal matrices of the 1000 beams were then obtained as

$$
\mathbf{K}^{(i)}=\Psi^{T} \mathbf{K}_{G}^{(i)} \Psi, \quad i=1, \ldots, 1000
$$

with the corresponding mass matrices equal to the 20x20 unit matrix. To validate the use of this modal basis, the natural frequencies obtained from the modal models were compared to those obtained directly from Nastran. For the first 3 frequencies (those considered of interest here), the means and standard deviations of the relative errors were all below $0.2 \%$ with the largest error over the $3 \times 1000$ set being $1.4 \%$ on a second natural frequency. These errors were considered small enough to warrant the use of the chosen basis.

As a starting point for the stochastic modeling of the matrices $\mathbf{K}^{(i)}$ as in Eq. (1), the matrices $\mathbf{K}_{0}$ and $\mathbf{K}_{1}$ for the straight beam, denoted in the sequel as $\hat{\mathbf{K}}_{0}$ and $\hat{\mathbf{K}}_{1}$ were evaluated next. Given the choice of modal basis, the matrix $\hat{\mathbf{K}}_{0}$ is diagonal with elements equal to the squared natural frequencies of 20 straight beam modes. The evaluation of the matrix $\hat{\mathbf{K}}_{1}$ was accomplished by imposing a small uniform temperature on the beam $\left(1.0^{\circ} \mathrm{C}\right)$, extracting the 
global stiffness matrix of the finite element model of the heated beam, projecting it on the basis as in Eq. (4), and forcing an equality of the matrices in Eq. (1) with the identified preload of $P=$ 22.1N. Note that the part of the matrix $\hat{\mathbf{K}}_{1}$ relevant to the transverse modes is positive definite as expected but the part associated to the in-plane modes is not due to the limited computational accuracy, i.e. the frequencies of these modes should not be affected by the preload. Since positive semi-definiteness is required for the proposed modeling, as described in the ensuing section, the

matrix $\hat{\mathbf{K}}_{1}$ was modified as follows. It was first decomposed into its eigenvectors and eigenvalues, the latter ones which are negative were then replaced by their absolute values, and the matrix was recomposed from these eigenvalues and the eigenvectors. The matrix denoted as $\hat{\mathbf{K}}_{1}$ in the ensuing discussion is the one resulting from this modification.

\section{MAXIMUM ENTROPY BASED NONPARAMETRIC MODELING}

It is proposed here to accomplish the stochastic modeling of the matrices $\mathbf{K}_{0}$ and $\mathbf{K}_{1}$ with the maximum entropy based nonparametric approach. In the context of random matrices, the approach is centered around the modeling of symmetric positive definite matrices of finite inverse (assuming that there are no rigid body modes). The maximization of the entropy under these constraints was carried out in [14] and leads to the representation of such random matrices $\mathbf{B}$ in the form

$$
\mathbf{B}=\overline{\mathbf{L}} \mathbf{H} \mathbf{H}^{T} \overline{\mathbf{L}}^{T}
$$

where $\overline{\mathbf{L}}$ is any decomposition, e.g. Cholesky, of the mean matrix $\overline{\mathbf{B}}$, i.e. satisfying $\overline{\mathbf{B}}=\overline{\mathbf{L}}^{T}$. Further, $\mathbf{H}$ denotes a lower triangular random matrix the elements of which are all statistically independent of each other. Moreover, 
(a) The diagonal elements $H_{i i}$ are obtained as $H_{i i}=\sqrt{\frac{Y_{i i}}{\mu}}$ where $Y_{i i}$ is Gamma distributed with parameter $(p(i)-1) / 2$, and

(b) the off-diagonal elements $H_{i l}, i \neq l$, are normally distributed (Gaussian) random variables with standard deviation $\sigma=1 / \sqrt{2 \mu}$ as pictorially described in Fig. 4. Note further that

$$
p(i)=n-i+2 \lambda-1 \quad \text { and } \quad \mu=\frac{n+2 \lambda-1}{2}
$$

with $n$ denoting the size of the matrices $\mathbf{B}$.

In the above equations, the parameter $\lambda>0$ is the free parameter of the statistical distribution of the random matrices $\mathbf{H}$ and $\mathbf{B}$ and can be evaluated to meet any given information about their variability, often the standard deviation of the lowest natural frequency of the random linear system will be specified and used to obtain $\lambda$. This condition, coupled with Eqs (5)-(7) and properties (a) and (b) provides a complete scheme for the generation of random symmetric positive definite matrices $\mathbf{B}$.

The entropy maximization-based formulation of the nonparametric approach permits the consideration of different or additional constraints as required by the problem at hand. This observation has led in particular to an extension [15] of the above, original methodology in which the standard deviation of several, $p<n$, natural frequencies are imposed in addition to the above constraints (symmetry, positive definiteness, finiteness of inverse). The resulting probabilistic model then exhibits more than the single parameter $\lambda$ for an improved matching of available data and shares many features of the one described above. Specifically, the corresponding random matrices $\mathbf{C}$ can be expressed as

$$
\mathbf{C}=\overline{\mathbf{D}} \tilde{\mathbf{H}} \tilde{\mathbf{H}}^{T} \overline{\mathbf{D}}^{T}
$$


where $\overline{\mathbf{D}}$ is a particular decomposition of the mean matrix $\overline{\mathbf{C}}$ satisfying $\overline{\mathbf{C}}=\overline{\mathbf{D}} \overline{\mathbf{D}}^{T}$, see [15]. The matrix $\widetilde{\mathbf{H}}$ is, as $\mathbf{H}$ in Eq. (5), lower triangular. Moreover, the elements of these two matrices are related to one another as

$$
\begin{gathered}
\tilde{H}_{i l}=\frac{H_{i l}}{\sqrt{G_{i i}}} \sqrt{\widetilde{G}_{i i}} \quad \text { for } l=1, \ldots, i \text { and } i=1, \ldots, p \\
\tilde{H}_{i l}=H_{i l} \quad \text { for } l=1, \ldots, i \text { and } i=p+1, \ldots, n
\end{gathered}
$$

where

$$
G_{i i}=\sum_{l=1}^{i} H_{i l}^{2}
$$

Finally, the random variables $\widetilde{G}_{i i}$ are independent of the above ones and of each other. They are distributed according to the probability density functions

$$
p_{\tilde{G}_{i i}}(g)=\bar{C}_{i} g^{(n+2 \lambda-3) / 2} \exp \left[-\mu_{i} g-\tau_{i} g^{2}\right] g \geq 0
$$

in which $\bar{C}_{i}$ are the appropriate normalization constants to ensure unit total probability and the parameters $\mu_{i}$ and $\tau_{i}$ must satisfy the mean condition

$$
E\left[G_{i i}\right]=1
$$

and the prescribed standard deviations of $p$ natural frequencies. Note finally that algorithms for the simulation of the samples $\tilde{G}_{i i}$ from the distribution of Eq. (11) are discussed in details in [15].

The above developments can also be relied on for the modeling of arbitrary random matrices $\mathbf{U}$ of mean $\overline{\mathbf{U}}$ using the decomposition (see [16])

$$
\overline{\mathbf{U}}=\overline{\mathbf{Q}} \overline{\mathbf{B}}
$$


where $\overline{\mathbf{B}}$ is the symmetric positive definite matrix such that

$$
\overline{\mathbf{B}}^{2}=\overline{\mathbf{U}}^{T} \overline{\mathbf{U}}
$$

and $\overline{\mathbf{Q}}$ is the unitary matrix (i.e., satisfying the property $\overline{\mathbf{Q}}^{T} \overline{\mathbf{Q}}=\mathbf{I}$, I denoting the identity matrix of appropriate dimension)

$$
\overline{\mathbf{Q}}=\overline{\mathbf{U}} \overline{\mathbf{B}}^{-1}
$$

Using either of the modeling procedures described above for symmetric positive definite matrices, random matrices $\mathbf{B}$ of mean $\overline{\mathbf{B}}$ are obtained to which correspond realizations of the matrix $\mathbf{U}$ as

$$
\mathbf{U}=\overline{\mathbf{Q}} \mathbf{B}
$$

Note that the matrix $\overline{\mathbf{Q}}$ is identity if the matrix $\overline{\mathbf{U}}$ is square, symmetric, and positive definite so that Eq. (16) simply reduces to the previous representation.

\section{PRELOAD PARAMETRIC MODELING}

It is proposed here to adopt a polynomial-chaos type representation of the preload, i.e. (e.g. see $[17,18])$

$$
P=\sum_{l=0} \gamma_{l} Q_{l}(V)
$$

where $V$ is a random variable of specified distribution, $Q_{l}(v)$ are appropriate polynomials in the variable $v$, and $\gamma_{l}$ are deterministic parameters. To accelerate the convergence of the series, the distribution of the random variable $V$ is selected according to the range of values exhibited by the preload (see discussion of [17]). For example, preload values exhibiting both negative and positive values without any particular upper or lower bound would suggest the use of a Gaussian 
random variable $V$ and to the selection of the Hermite polynomials for $Q_{l}(v)$. However, purely positive values of $P$ would lead to a Gamma modeling of the random variable $V$ and the use of the Laguerre polynomials for $Q_{l}(v)$.

The combination of the maximum entropy based nonparametric approach, for $\mathbf{K}_{0}$ and/or $\mathbf{K}_{1}$, and a polynomial chaos representation, for the preload $P$, proposed here is along the same perspective as the generalized stochastic modeling introduced recently in [19].

\section{MEASUREMENTS AND MAXIMUM LIKELIHOOD PARAMETER ESTIMATION}

The stochastic model of Eq. (1) involves a series of deterministic parameters that characterize it, including in particular the parameters $\gamma_{l}$ specifying the distribution of the random preload $P$ as well as the value of $\lambda$ for the matrices $\mathbf{K}_{0}$ and/or $\mathbf{K}_{1}$. If the extended methodology of Eqs (8)-(12) is adopted, the description of these matrices also requires the determination of the coefficients $\mu_{i}$ or $\tau_{i}$, one of these two sets of coefficients being evaluated from the mean condition of Eq. (12). In addition to these parameters characterizing mostly the variability of the beam stiffness matrix, it may also be necessary to update the mean matrices $\overline{\mathbf{K}}_{0}$ and $\overline{\mathbf{K}}_{1}$ which may deviate from their straight beam counterparts $\hat{\mathbf{K}}_{0}$ and $\hat{\mathbf{K}}_{1}$ owing to a possible asymmetry induced by the randomness of the beam curved profiles.

These various parameters, regrouped in the vector $\underline{\theta}$, will be estimated in a maximum likelihood framework from measurements of the modal characteristics of the beams. These include the first three random natural frequencies $\Omega_{j}, j=1,2,3$, of observed values $\omega_{j}^{(i)}$, and, potentially, measurements $\underline{\phi}_{j}^{(i)}$ of the corresponding mode shapes $\underline{\Phi}_{j}$. This mode shape 
information is first reduced by projection on the straight beam modal basis yielding the random projection coefficients $A_{j k}$ and their realized values $\alpha_{j k}$ satisfying

$$
\underline{\Phi}_{j}=\sum_{k} A_{j k} \underline{\psi}_{k} \quad \text { or } \quad A_{j k}=\underline{\psi}_{k}^{T} \mathbf{M}_{G} \underline{\Phi}_{j}
$$

and similarly for the observed mode shapes

$$
\underline{\phi}_{j}^{(i)}=\sum_{k} \alpha_{j k}^{(i)} \underline{\psi}_{k} \quad \text { or } \quad \alpha_{j k}^{(i)}=\underline{\psi}_{k}^{T} \mathbf{M}_{G} \underline{\phi}_{j}^{(i)}
$$

owing to the orthogonality of the straight beam modes with respect to $\mathbf{M}_{G}$, the finite element mass matrix of the straight beam.

Assuming that there is no randomness on the finite element mass matrix of the beams (the effect of the curvature is very small), the random mode shapes $\underline{\Phi}_{j}$ satisfy the orthogonality properties

$$
\underline{\Phi}_{k}^{T} \mathbf{M}_{G} \underline{\Phi}_{j}=0 \text { for } j \neq k
$$

as well as a normalization condition such as

$$
\underline{\Phi}_{j}^{T} \mathbf{M}_{G} \underline{\Phi}_{j}=1
$$

The existence of such conditions imply that the coefficients $A_{j k}$ must be related to one another. In fact, one can show that of the $m^{2}$ values $A_{j k}, j, k=1, \ldots, m, m$ being the number of modes considered, i.e. $m=3$ here, only $m(m-1) / 2$ form an independent set. This observation has led (see [20]) to further reduce the modal information to the skew coefficients

$$
B_{j k}=\left(A_{j k}-A_{k j}\right) / 2 \quad k>j
$$

and their realized values

$$
\beta_{j k}=\left(\alpha_{j k}-\alpha_{k j}\right) / 2 \quad k>j .
$$


The random vector of measurements $\underline{X}$ considered here is thus $\underline{X}=\left[\Omega_{1} \Omega_{2} \Omega_{3}\right]$ when only the frequencies are observed or $\underline{X}=\left[\Omega_{1} \Omega_{2} \Omega_{3} B_{12} B_{13} B_{23}\right]$ when the mode shapes are also captured. The vector of actual measurements for beam $i$ will consistently be denoted as $\underline{x}^{(i)}$.

Adopting the maximum likelihood framework, the deterministic parameters of the model stacked in the vector $\underline{\theta}$ will be chosen to maximize the likelihood function

$$
L=\prod_{i=1}^{M} p_{\underline{X}}\left(\underline{x}^{(i)} ; \underline{\theta}\right)
$$

where $p_{\underline{X}}(\underline{x} ; \underline{\theta})$ denotes the probability density function of the random vector $\underline{X}$. Two approaches will be considered for the estimation of this probability density function, the first one of which is the joint Gaussian approximation

$$
p_{\underline{X}}^{G}(\underline{x} ; \underline{\theta})=\frac{1}{(2 \pi)^{p / 2} \sqrt{\operatorname{det}\left(K_{\underline{X} \underline{X}}\right)}} \exp \left[-\frac{1}{2}\left(\underline{x}-\underline{\mu}_{\underline{X}}\right)^{T} K_{\underline{X} \underline{X}}^{-1}\left(\underline{\underline{x}}-\underline{\mu}_{\underline{X}}\right)\right]
$$

where $p=3$ or 6 is the number of components of $\underline{X}$ and $\underline{\mu}_{\underline{X}}(\underline{\theta})$ and $K_{\underline{X}} \underline{X}(\underline{\theta})$ denote the mean vector and covariance matrix of the random vector $\underline{X}$ determined from the model of Eq. (1) with the parameter values in $\underline{\theta}$.

A more refined estimate of $p_{\underline{X}}(\underline{x} ; \underline{\theta})$ can be obtained by the kernel density estimation technique (see [21,22], for theoretical discussions and [23] for an application). According to this methodology, the probability density function is estimated as

$$
p_{\underline{X}}^{K}(\underline{x} ; \underline{\theta})=\frac{1}{M} \sum_{i=1}^{M} \prod_{l=1}^{p}\left\{\frac{1}{h_{l}} \mathrm{~K}\left(\frac{y_{l}^{(i)}-y_{l}}{h_{l}}\right)\right\}
$$

where $y_{l}$ and $y_{l}^{(i)}$ are the lth component of the vectors $\underline{y}$ and $\underline{y}^{(i)}$ defined as 


$$
\underline{y}=\Xi^{T}\left(\underline{x}-\underline{\mu}_{\underline{X}}\right) \quad \text { and } \quad \underline{y}^{(i)}=\Xi^{T}\left(\underline{x}^{(i)}-\underline{\mu}_{\underline{X}}\right)
$$

in which $\Xi$ is the matrix obtained by stacking by columns the normalized eigenvectors of the

covariance matrix $K_{\underline{X} X}(\underline{\theta})$. Further, $\mathrm{K}(\cdot)$ denotes the kernel and $h_{l}$ denotes coefficients related to it. For the Gaussian kernel, one has

$$
\mathrm{K}(u)=\frac{1}{\sqrt{2 \pi}} \exp \left(-u^{2} / 2\right) \quad ; h_{l}=\sqrt{\Lambda_{l l}}\left[\frac{4}{M(2+p)}\right]^{1 /(4+p)}
$$

in which $\Lambda_{l l}$ is the lth eigenvalue of $K_{\underline{X} \underline{X}}(\underline{\theta})$.

\section{NUMERICAL RESULTS}

The above sections have presented a general framework for the representation of modal data, natural frequencies and/or mode shape information, according to the model of Eq. (1). A first validation of these concepts is presented here for the computational clamping data of option A, see Figs 2(a) and 3(a) for the scatter plots of the frequencies.

As stated earrlier, it is observed from these figures that the natural frequencies occupy a wedge shaped domain as opposed to forming a narrow curve as seen in [12] on the data of [11]. It is thus concluded that either or both matrices $\mathbf{K}_{0}$ and/or $\mathbf{K}_{1}$ must be considered random in addition to the preload $P$. Further, the tip of the wedge, i.e. the lowest natural frequencies, closely corresponds to the straight beam values confirming the almost exclusively positive values of the preload. Further, the sharpness of the wedge indicates that very little variability does exist in the frequencies when the preload value is close to zero. This finding suggests that the matrix $\mathbf{K}_{0}$ should not be randomized here and should be close to its straight beam counterpart $\hat{\mathbf{K}}_{0}$. 
On the basis of these observations, the model of Eq. (1) will be sought with $\mathbf{K}_{1}$ alone represented by the nonparametric approach and the preload $P$ will be expressed in terms of a Gamma distributed random variable $V$ of distribution

$$
p_{V}(v)=\frac{1}{\Gamma(a)} v^{a-1} e^{-v} \quad v \in[0, \infty) \text { and } a \geq 0
$$

where $\Gamma(\cdot)$ denotes the Gamma function. In performing the maximum likelihood identification, it was found very valuable to start with a simple model and grow its complexity as necessary using as initial conditions for the optimization process (carried out here with fminsearch in matlab) the converged values obtained with a simpler model. Such an approach was also adopted in regards to the probability density function representation, i.e. initially with the Gaussian approximation of Eq. (25) and then with its kernel counterpart of Eq. (26). In this light, the nonparametric model of Eq. (5)-(7) was first adopted for the matrix $\mathbf{K}_{1}$ and a two term representation of the preload $P$, Eq. (17), was selected. This model has 4 uncertainty-related parameters: the two parameters $\gamma_{0}$ and $\gamma_{1}$, the shape parameter $a$, and the nonparametric coefficient $\lambda$. In addition to these 4 parameters, the first three diagonal elements of the constant matrix $\mathbf{K}_{0}$ and of the mean matrix $\overline{\mathbf{K}}_{1}$ were also identified. In all, the parameter vector $\underline{\theta}$ included 10 components all identified by the maximum likelihood approach.

Modal tests can provide both natural frequencies and mode shape data but the former are much easier to gather than the latter (only one sensor is needed for example). It was accordingly proposed to use only the natural frequencies data (the first three) in the identification process of the model parameters but to use the mode shape information as a mean to assess the validity of the model of Eq. (1) once the best parameter values were obtained. As a first check, the identification process led to values of the diagonal elements of $\mathbf{K}_{0}$ that were very close from 
those of $\hat{\mathbf{K}}_{0}$, as expected from the above discussion. However, the diagonal elements of $\overline{\mathbf{K}}_{1}$ did not match those of $\hat{\mathbf{K}}_{1}$ demonstrating the importance of identifying the mean model as well as its uncertainty related parameters. The parameters $\gamma_{0}, \gamma_{1}$, the shape parameter $a$, and the nonparametric coefficient $\lambda$ were identified as: $12.1,-11.0,1.17$, and 15.4 .

Then, shown in Fig. 5 are the probability density functions of the first three natural frequencies as obtained from the computational experiment and from the model of Eq. (1) while in Fig. 6 are the corresponding joint probability density functions of the first two natural frequencies. The good matching of these curves suggests indeed the applicability of the model of Eq. (1). As discussed above, the probability density functions of the modal coefficients $\beta_{21}$, $\beta_{31}$, and $\beta_{32}$, see Eq. (23), were also compared and are shown in Figs 7 . In assessing the matching of these curves, note that the experimental modal coefficients were not used in the identification and are not strongly correlated to the frequencies used in the identification (the largest such coefficient of correlation is 0.27 ). Thus, the good to very good match of the curves in Fig. 7 further strengthen the validation of the model of Eq. (1) for the representation of the modal properties of preloaded structures. A similar comparison was finally made between the probability density functions of the preloads, see Fig. 8, which again match very well even though this information was also not used in the identification.

Since Eqs (1) and (17) led to a good matching of the computational experiment data, it was decided to use this model to quantify the importance on the natural frequencies of the uncertainties in the preload and in the matrix $\mathbf{K}_{1}$. For this purpose, two additional runs were carried out, one in which the preload was assumed to remain equal to its mean value while in the other it was the matrix $\mathbf{K}_{1}$ which was not randomized. The coefficients of variations of the three 
natural frequencies obtained in the former case were very close to $1 / 2$ of those induced by the variability of the preload alone. This observation confirms the importance of modeling the preload randomly but also demonstrate that the variability in the resulting clamped geometry may also have a significant effect.

\section{CONCLUSIONS}

This paper addresses the stochastic modeling of the stiffness matrix of slender uncertain curved beams forced fit into a clamped-clamped fixture designed for straight beams as an example of structures preloaded during assembly.

Central to the modeling is the explicit introduction of a random preload $P$ multiplying a random preload-dependent stiffness matrix $\mathbf{K}_{1}$. This preload-related component is then added to the preload-independent stiffness matrix $\mathbf{K}_{0}$, see Eq. (1). A parametric stochastic modeling of the preload $P$ was introduced while a nonparametric maximum entropy-based representation of the random matrices $\mathbf{K}_{0}$ and/or $\mathbf{K}_{1}$ was suggested. Finally, a maximum likelihood framework was proposed for the identification of the parameters associated with the uncertainty level and the mean model, especially the mean of the matrix $\mathbf{K}_{1}$, using either natural frequencies only or natural frequencies and mode shape information of the structure after assembly.

To validate these concepts, a simulated, computational experiment was conducted within Nastran to produce a population of natural frequencies and mode shapes of uncertain slender curved beams after clamping. The application of the above concepts to this simulated data led to a good to very good matching of the probability density functions of the natural frequencies and the modal components, even though this information was not used in the identification process. These results strongly suggest the applicability of the proposed stochastic model. 


\section{ACKNOWLEDGEMENTS}

The authors are grateful to one of the reviewers whose comments and suggestions helped strengthened the paper.

\section{REFERENCES}

[1] R.A. Ibrahim, C.L. Pettit, Uncertainties and dynamic problems of bolted joints and other fasteners, Journal of Sound and Vibration 279 (2005) 857-936.

[2] F. Dohnal, B.R. Mace, N.S. Ferguson, Joint uncertainty propagation in linear structural dynamics using stochastic reduced basis methods, AIAA Journal 47 (2009) 961-969.

[3] D.J. Segalman, D.L. Gregory, M.J. Starr, B.R. Resor, M.D. Jew, J.P. Lauffer, N.M. Ames, Handbook on Dynamics of Jointed Structures, Sandia Report SAND2009-4164, 2009.

[4] C. Blanze, P. Rouch, Analysis of structures with stochastic interfaces in the mediumfrequency range, Journal of Computational Acoustics 13 (2005) 711-729.

[5] B. Huang, Q.S. Li, W.H. Shi, Z. Wu, Eigenvalues of structures with uncertain elastic boundary restraints, Applied Acoustics 68 (2007) 350-363.

[6] J. Avalos, M.P. Mignolet, C. Soize, Response of bladed disks with mistuned blade-disk interface,” Proceedings of the IGTI Turbo Expo’09, Orlando, Florida, June 2009, ASME Paper GT-2009-59580.

[7] M.P. Mignolet, C. Soize, J. Avalos, Nonparametric Stochastic Modeling of Structures with Uncertain Boundary Conditions/Coupling Between Substructures, AIAA Journal 51 (2013) 1296-1308. 
[8] C. Soize, Stochastic Models of Uncertainties in Computational Mechanics, American Society of Civil Engineers (ASCE), Reston, 2012.

[9] H. Chebli, C. Soize, Experimental validation of a nonparametric probabilistic model of nonhomogeneous uncertainties for dynamical systems, Journal of the Acoustical Society of America 115 (2004) 697-705.

[10] J. Duchereau, C. Soize, Transient dynamics in structures with non-homogeneous uncertainties induced by complex joints, Mechanical Systems and Signal Processing 20 (2006) 854-867.

[11] R. Murthy, X.Q. Wang, R. Perez, M.P. Mignolet, L.A. Richter, Uncertainty-based experimental validation of nonlinear reduced order models, Journal of Sound and Vibration 331 (2012) 1097-1114.

[12] A. Shukla, M.P. Mignolet, M.P., Identification and updating of uncertain dynamic models of a flat beam for nonlinear forced response predictions, Proceedings of the 52nd Structures, Structural Dynamics and Materials Conference, Denver, Colorado, April 2011, AIAA Paper AIAA 2011-2082.

[13] R.W. Gordon, J.J. Hollkamp, S.M. Spottswood, Nonlinear response of a clamped-clamped beam to random base excitation,” Proceedings of the Eighth International Conference on Recent Advances in Structural Dynamics, Southampton, England, 2003.

[14] C. Soize, A nonparametric model of random uncertainties on reduced matrix model in structural dynamics, Probabilistic Engineering Mechanics 15 (200) 277-294.

[15] M.P. Mignolet, C. Soize, Nonparametric stochastic modeling of linear systems with prescribed variance of several natural frequencies, Probabilistic Engineering Mechanics 23 (2008) 267-278. 
[16] C. Soize, Random matrix theory for modeling uncertainties in computational mechanics, Computer Methods in Applied Mechanics and Engineering 194 (2005) 1333-1366.

[17] C. Soize, R. Ghanem, Physical systems with random uncertainties : chaos representation with arbitrary probability measure, SIAM Journal on Scientific Computing, 26 (2004) 395410.

[18] S. Sakamoto, R. Ghanem, Polynomial chaos decomposition for the simulation of nonGaussian nonstationary stochastic processes, Journal of Engineering Mechanics 128 (2002) 190-201.

[19] C. Soize, Generalized probabilistic approach of uncertainties in computational dynamics using random matrices and polynomial chaos decompositions, International Journal for Numerical Methods in Engineering, 81 (2010) 939-979.

[20] J. Avalos, E.D. Swenson, M.P. Mignolet, N.J. Lindsley, Stochastic modeling of structural uncertainty/variability from GVT test data, Journal of Aircraft 49 (2012) 870-884.

[21] A.W. Bowman, A. Azzalini, Applied smoothing techniques for data analysis, Oxford University Press, 1997.

[22] G.R. Terrell, D.W. Scott, Variable kernel density estimation, The Annals of Statistics 20 (1992) 1236-1265.

[23] C. Soize, A computational inverse method for identification of non-Gaussian random fields using the Bayesian approach in very high dimension, Computer Methods in Applied Mechanics and Engineering 200 (2011) 3083-3099. 


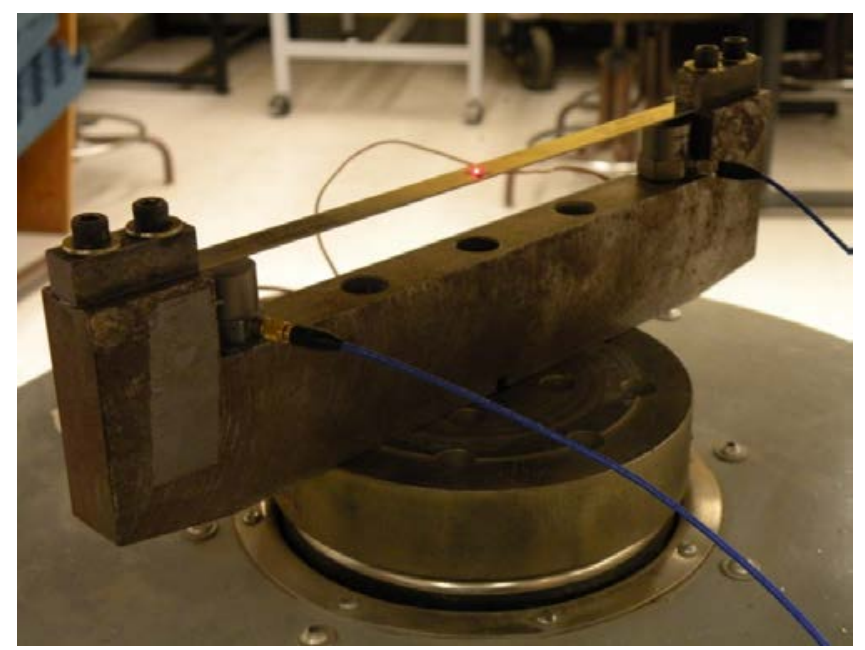

(a)
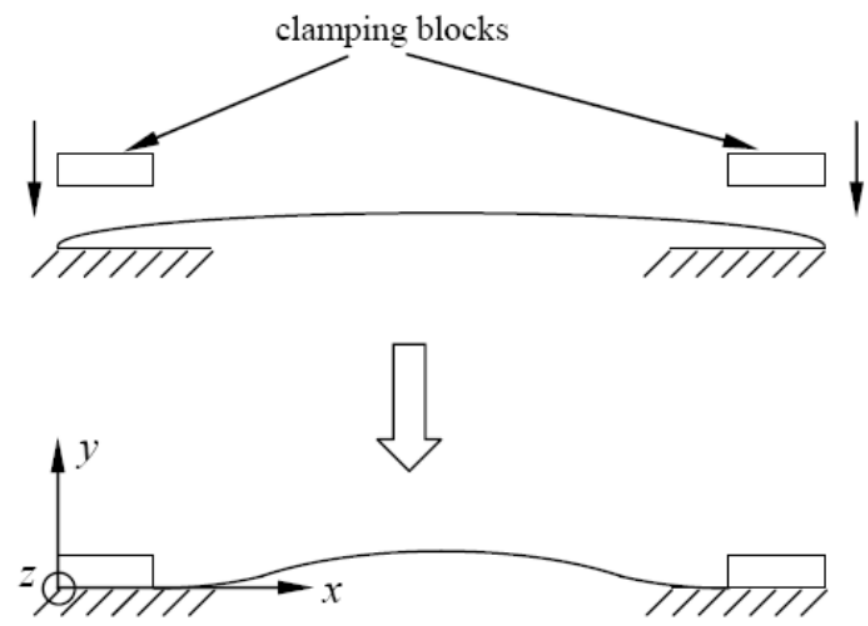

(b)

Figure 1. (a) Clamping fixture and beam in the physical experiment. (b) Clamping process in the computational experiment. 

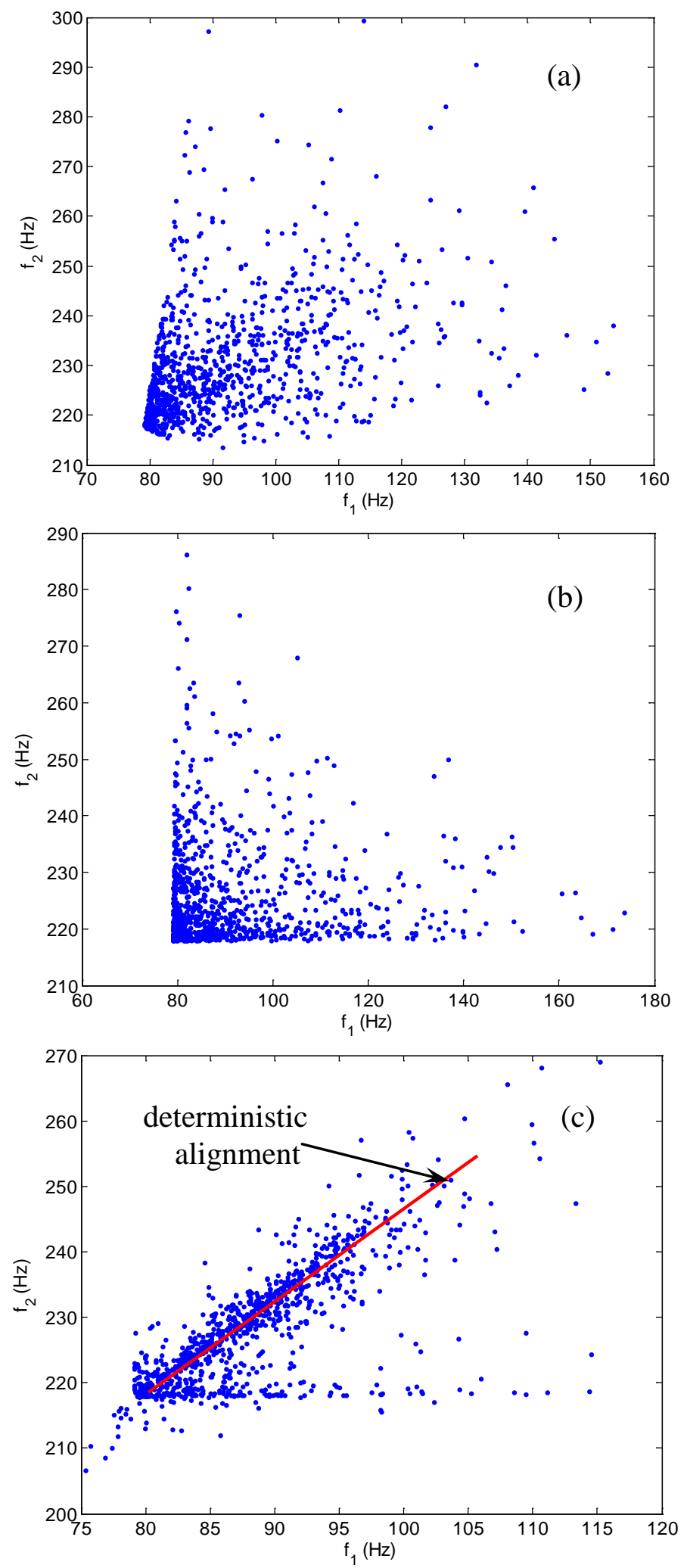

Figure 2. Scatter plot of first and second natural frequencies obtained from the computational experiment according to the three clamping options (a) A, (b) B, (c) C. 

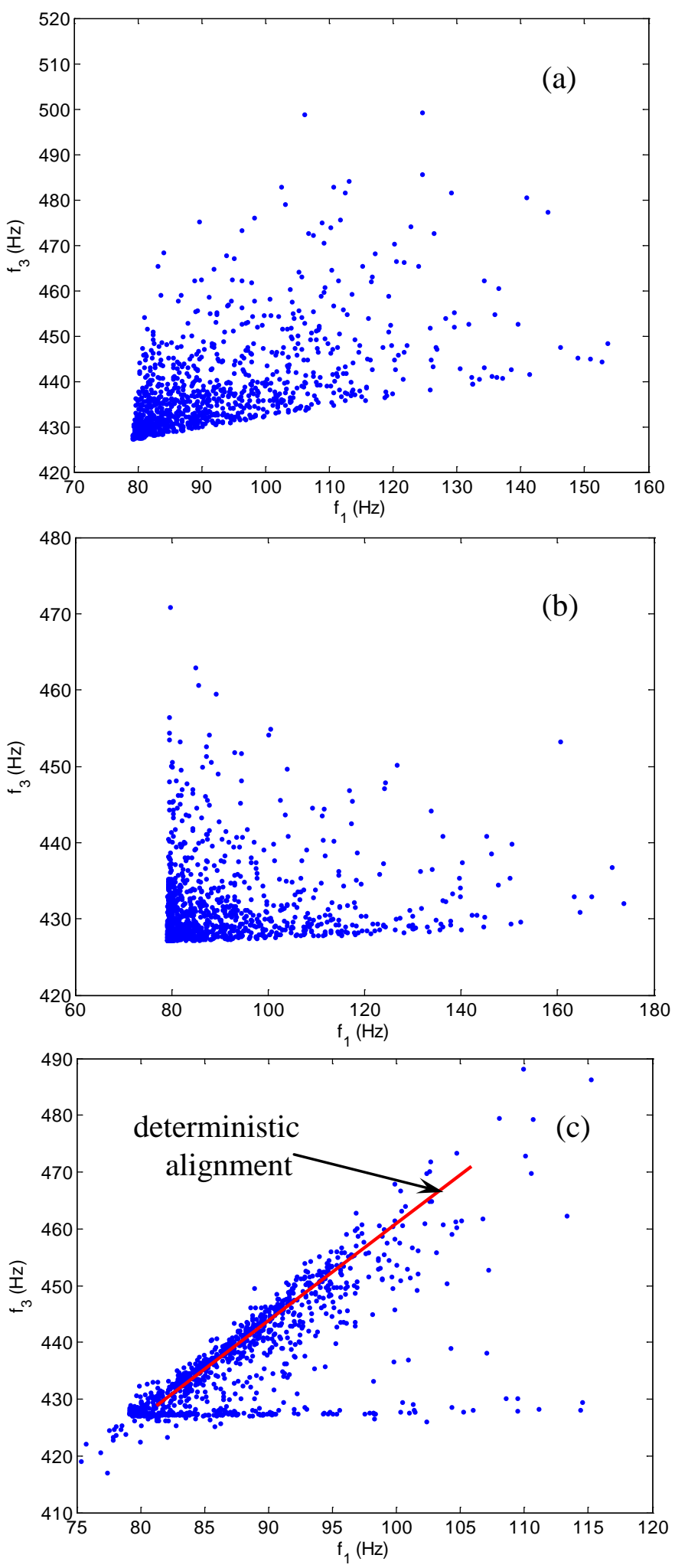

Figure 3. Scatter plot of first and third natural frequencies obtained from the computational experiment according to the three clamping options (a) A, (b) B, (c) C. 


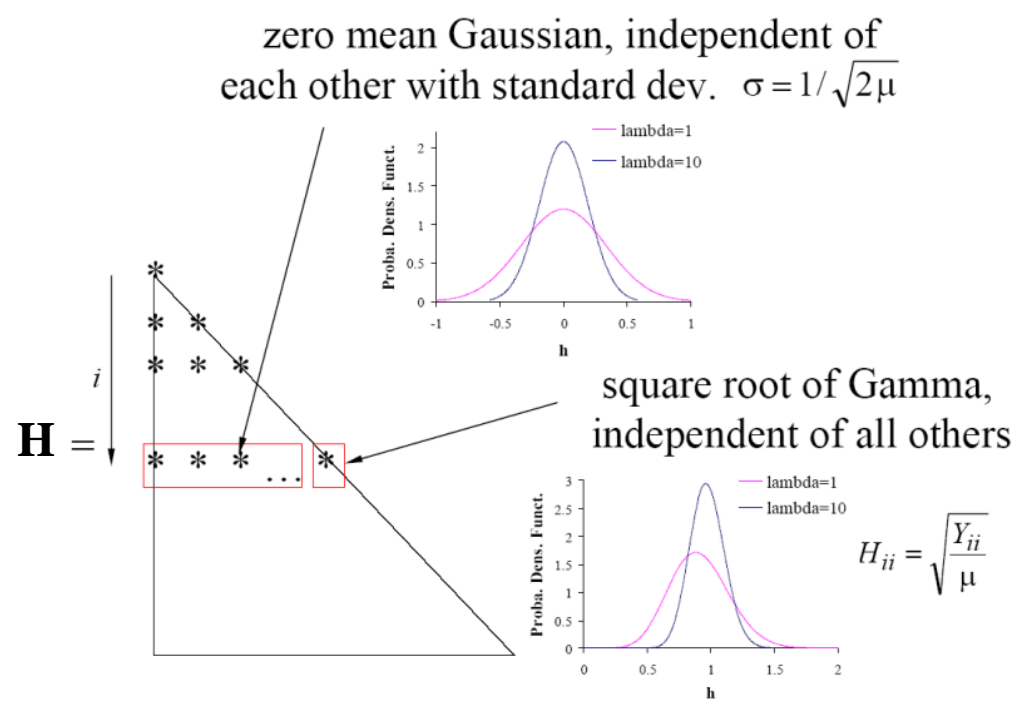

Figure 4. Structure of the random $\mathrm{H}$ matrices with $n=8, i=2$, and $\lambda=1$ and 10 . 

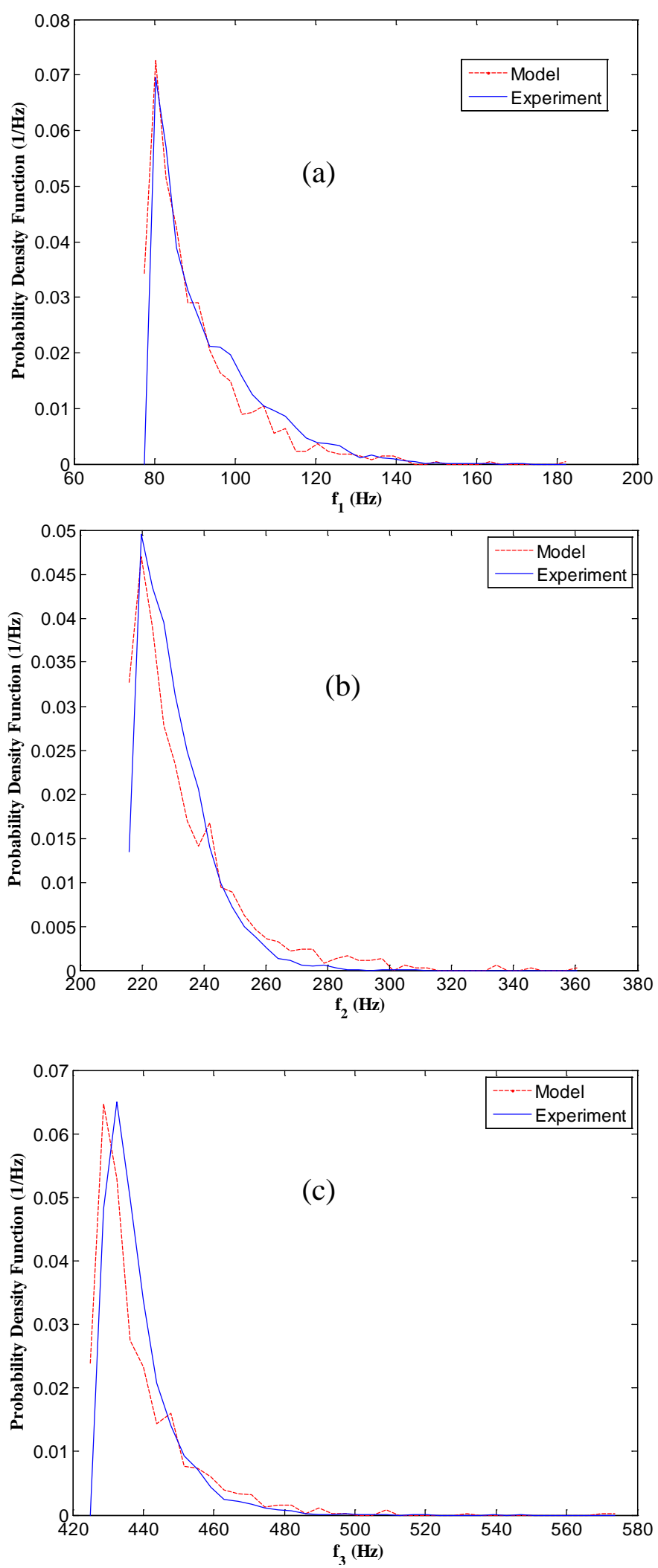

Figure 5. Probability density functions of the (a) first, (b) second, and (c) third natural frequency. Computational experiment with Eq. (3) and model of Eq. (1) with maximum likelihood identified parameters. 


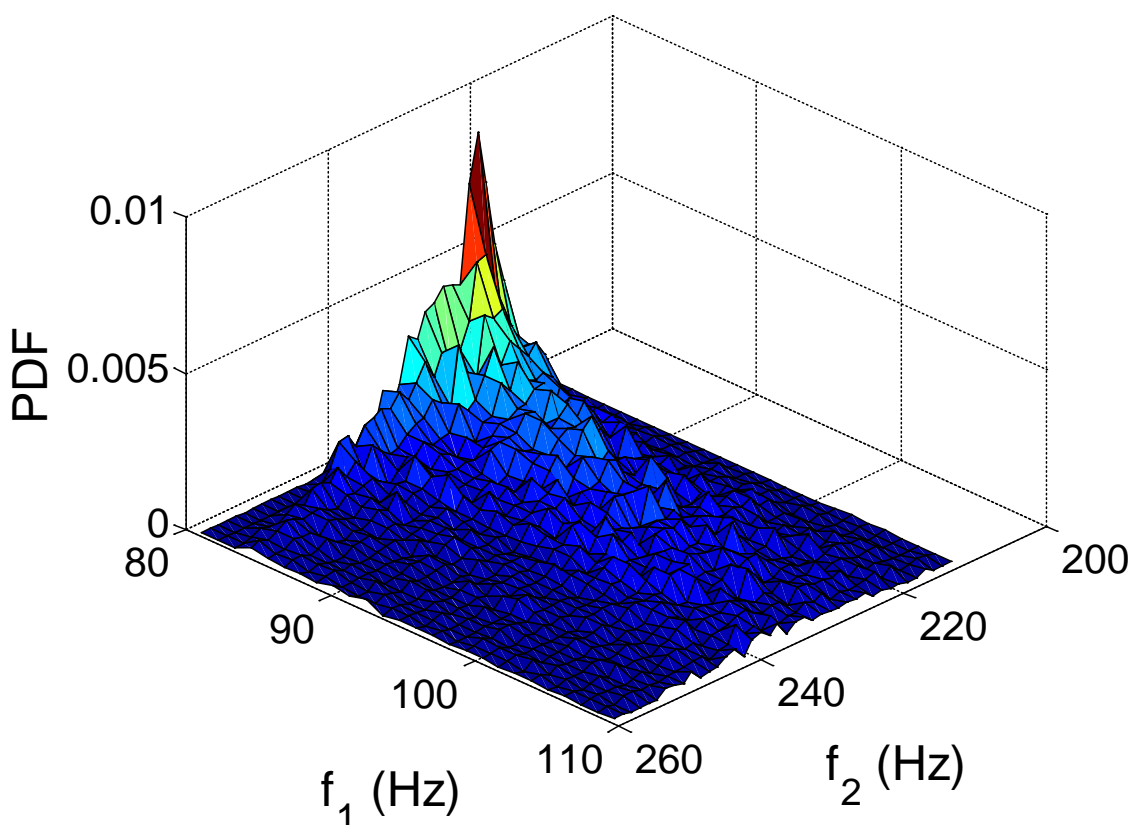

(a)

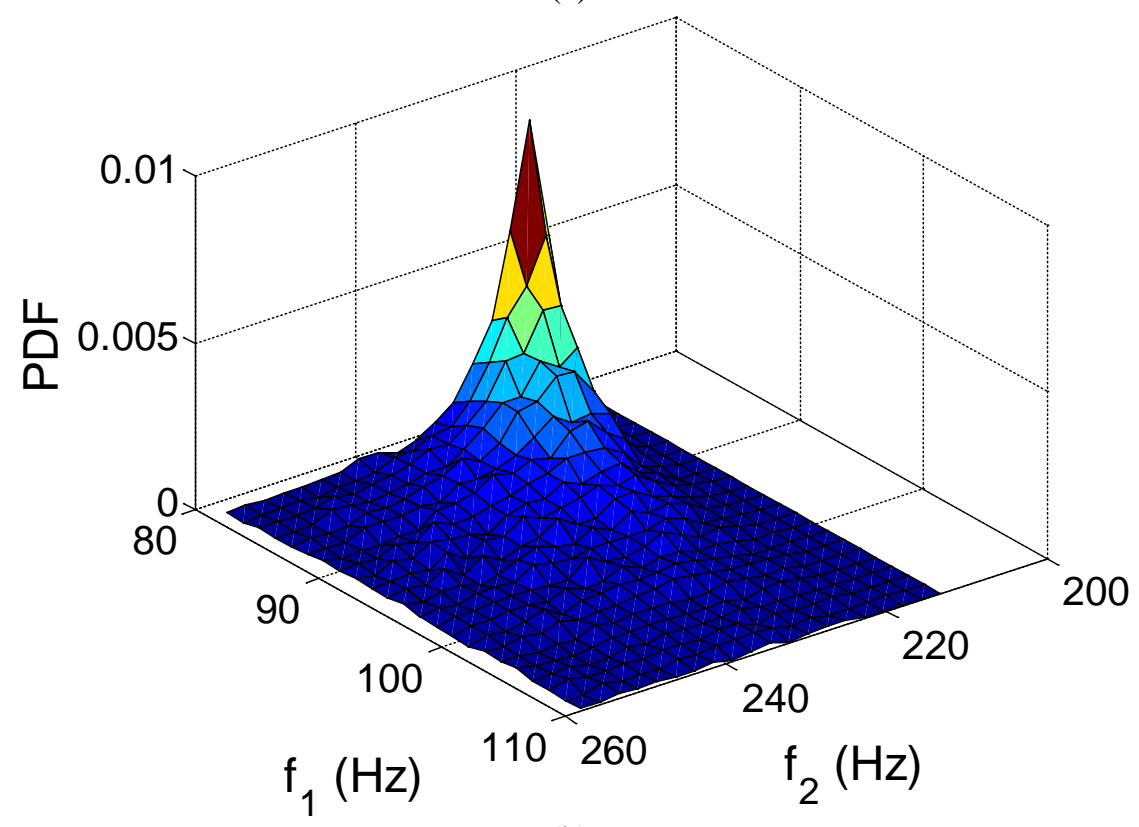

(b)

Figure 6. Joint probability density functions of the first and second natural frequencies. (a) Computational experiment with Eq. (3) and (b) model of Eq. (1) with maximum likelihood identified parameters. 

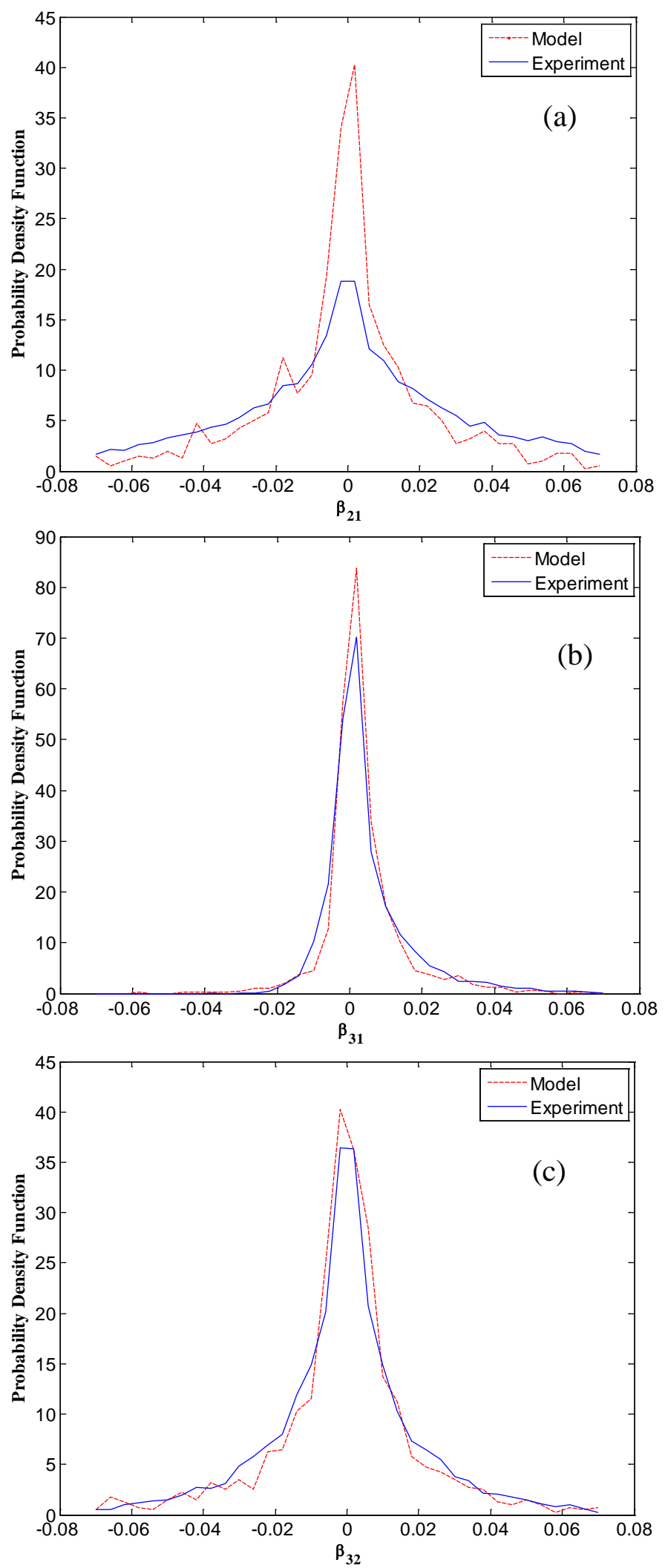

Figure 7. Probability density functions of the modal coefficients (a) $\beta_{21}$, (b) $\beta_{31}$, and (c) $\beta_{32}$. Computational experiment with Eq. (3) and model of Eq. (1) with maximum likelihood identified parameters. 


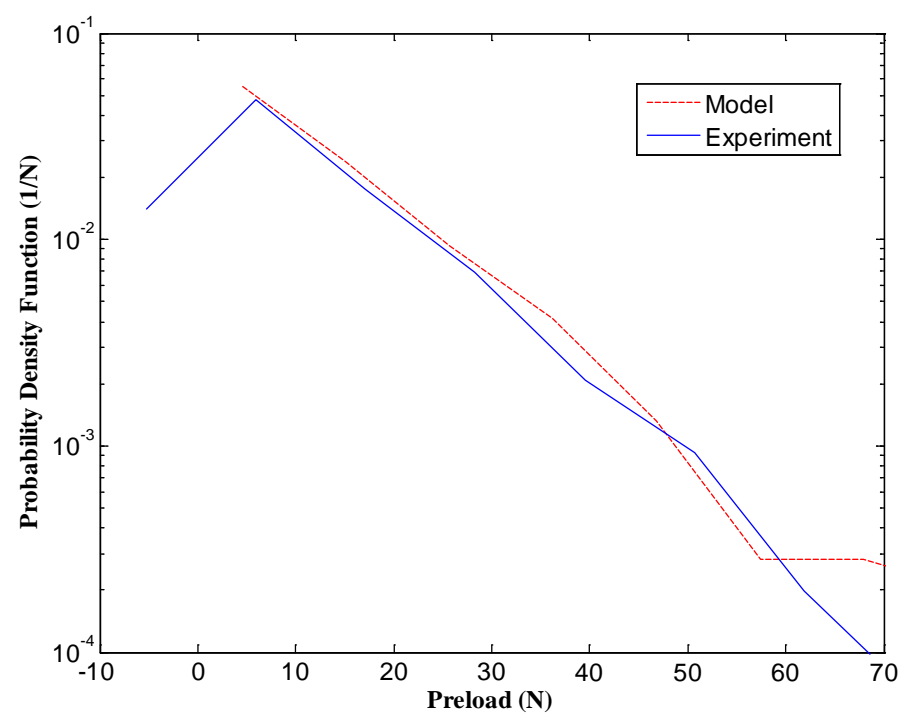

Figure 8. Probability density functions of the preload. Computational experiment with Eq. (3) and model of Eq. (1) with maximum likelihood identified parameters. 